\title{
Objective measurement of physical activity in a random sample of Saint-Petersburg inhabitants
}

\author{
Alexander Orlov ${ }^{1}$, Oxana Rotar $\odot^{1}$, Matthaeus Vigl ${ }^{2}$, Alexandra Konradi ${ }^{1}$, Heiner Boeing ${ }^{2}$ \\ ${ }^{1}$ Research Laboratory of Epidemiology of Noncommunicable Diseases, Almazov National Medical Research Center, Saint Petersburg, Russia \\ ${ }^{2}$ Department of Epidemiology, German Institute of Human Nutrition, Potsdam Rehbruecke, Germany
}

\begin{abstract}
Background: World Health Organization (WHO) experts listed physical inactivity in leading risk factors for global mortality. Current research shows that only objective measurement of physical activity may provide accurate information on this parameter. The aim of our study was to assess the 7-day physical activity monitoring using triaxial accelerometers in a random sample of Saint-Petersburg inhabitants.

Material and methods: As a part of all-Russian epidemiology survey ESSE-RF there was involved random sampling of 1600 Saint-Petersburg inhabitants (25-65 years) stratified by age and sex. After that a random sub-population of 100 subjects was selected. All subjects filled in questionnaire regarding physical activity, occupation, education and nutrition. Anthropometry (weight, height with body-mass index calculation, waist circumference) was performed. Actigraph GT3X+ (Actigraph LLC, USA) accelerometer and physical activity diary were used in order to evaluate physical activity monitoring for 7 days. Adequate levels of physical activity (PA) were defined as more than 10000 steps/day and at least 150 minutes/week of moderate and vigorous physical activity (MVPA) in bouts of 10 minutes or more.

Results: $1 / 2$ of subjects were physically active according to steps, and $1 / 3$ according to MVPA time criteria. No gender, occupation or body composition differences were revealed in physically active and inactive subjects. Almost $50 \%$ of physically active subjects had balanced workweek-weekend PA profile, and the same criterion is true only for $13 \%$ of subjects in inactive group. In both groups the same peaks of MVPA were revealed - at 8.00-9.00 and $18.00-19.00$, which are typical transportation time, but in active group these peaks were significantly higher. According to PA diaries, in most of cases physical inactivity was related to the usage of private or public transport.

Conclusion: Triaxial PA-monitoring shows, that $40-60 \%$ of subjects were physically inactive, and 150-min MVPA goal can easily be achieved by only increasing walking time during transportation peaks. The physical inactivity was not determined by the type of occupation, sex or age, instead it was mainly influenced by the usage of cars in the morning and evening transportation time, rather than walking.
\end{abstract}

Key words: physical activity level; hypodynamia; triaxial accelerometer; objective measurement

Arterial Hypertens. 2020, vol. 24, no. 3, pages: 135-141 DOI: 10.5603/AH.a2020.0018

Address for correspondence: Alexander Orlov, Research Laboratory of Epidemiology of Noncommunicable Diseases,

Almazov National Medical Research Center, Saint Petersburg, Russia;

e-mail: alexanderorlov1987@gmail.com

VM Copyright (C) 2020 Via Medica, ISSN 2449-6170

www.ah.viamedica.pl 


\section{Introduction}

Level of physical inactivity is rising in many countries with possible major implications for the general health of people and for the prevalence of noncommunicable diseases (NCD) such as cardiovascular diseases, diabetes, cancer and related risk factors (elevated blood pressure, impaired glucose metabolism, overweight). World Health Organization (WHO) experts identified physical inactivity as the fourth leading risk factor for global mortality [1]. Physical inactivity is estimated to be the principal cause for approximately $21-25 \%$ of breast and colon cancer burden, $27 \%$ of diabetes and approximately $30 \%$ of ischemic heart disease burden [1]. According to literature review [2-4], there is a dose-response relation between intensity, frequency, duration and volume of physical activity (PA) and NCD risk. World Health Organization recommendations indicate that 150 minutes per week of moderate- to vigorous-intensity physical activity (MVPA), accumulated in short multiple bouts of at least 10 minutes, or one long bout may lead to NCD reduction. Recent studies showed, that use of accelerometers may provide accurate information on not only PA related energy expenditure and PA intensity, but also may objectively calculate consecutive minutes in MVPA during leisure time activity, which is almost impossible with PA self-reports.

Although some studies including objective PA-measurement were performed in Russia, most of them used pedometers and none of them used triaxial accelerometers in a random population-based sample. It was shown that waist-mounted, uniaxial accelerometer, with cut points based on walking, misses some physical activity and triaxial devices tend to have higher correlations with energy expenditure [5].

The aim of our study was to assess a 7-day physical activity monitoring using triaxial accelerometers in a random sample of Saint-Petersburg inhabitants.

\section{Material and methods}

As a part of the nationwide Russian epidemiologic survey ESSE-RF a random sampling of 1600 SaintPetersburg inhabitants (25-65 years) stratified by age and sex was involved. A random sub-population of 100 subjects was subtracted from the initial sample, according to the age and sex distribution. On the day of recruitment, participants underwent anthropometric, behavioral and biochemical assessments, including fasting lipids and glucose levels (ARCHITECT (USA), Abbott). Socio-economic (including education and occupation), behavioral attributes as well as information on medical conditions and therapy were assessed using questionnaires administered by trained interviewers who utilized standard protocols (which were described before [6]). The trained personnel conducted height, weight, waist circumference and resting blood pressure measurements according to standard protocols.

For the physical activity monitoring all subjects were asked to wear an Actigraph GT3X+ (Actigraph LLC, USA) accelerometer for 7 days over the right hip on an elastic belt while they were awake and on the right wrist during sleep time. Participants were also asked to fill in a 7-days diary regarding their physical activity during time of investigation. Data were recorded with $100 \mathrm{~Hz}$ on three axes and then aggregated to 10-second epochs. Accelerometer data were obtained from 100 individuals. A valid measurement was defined as having at least 5 days with a minimum of 10 hours monitor wear. Wear time was determined by subtracting non-wear time from 24 hours. Non-wear was defined as zero activity intensity counts for 1 minute, or 0-99 counts for maximum 2 minutes. The amount of physical activity measured by accelerometer was presented according to number of steps and by the time spent in moderate and vigorous physical activity. Time spent in physical activity of moderate or vigorous intensity, separately or combined, was based on application of count thresholds corresponding to moderate- or vigorous-intensity activity. Intensity-threshold criteria were 1952-5724 counts for moderate intensity (equivalent to 3 METs) and 5725-9498 counts for vigorous intensity (6 METs). Time spent in activity of a defined intensity (moderate, vigorous, or moderate and vigorous combined) was determined by summing up minutes of a day where the count met the criterion for the respective intensity. Moderate to vigorous physical activity (MVPA) duration data was also presented in sustained bouts, in accordance with physical activity recommendations, where 10-min MVPA bouts were defined as 10 or more consecutive minutes above the relevant threshold. Mean daily time in bouts was calculated across all valid days.

Adequate levels of physical activity were defined in 3 ways - according to number of steps - more then 10000 steps/day; according to time, spent in moderate and vigorous physical activity - more than 300 minutes/week; according to time of moderate and vigorous physical activity in bouts of $10-\mathrm{min}$ utes or more - at least 150 minutes/week [7].

Physical activity was defined as balanced when participants spent $20-35 \%$ of their MVPA time in 10 -min bouts on weekend (Saturday and Sunday); 
less than 20\% of MVPA on the weekend was defined as main physical activity during workweek (from Monday to Friday), and more than 35\% of MVPA on the weekend as main physical activity on weekend.

The definition of occupational categories was performed, according to the data from the questionnaire. Subjects with intellectual type of occupation were defined as "intellectual group", manual workers were defined as "manual group", and other subjects (including retired participants) were defined as "average group".

Statistical analysis was performed by statistical package SSPS Statistics 20 (IBM, USA). The sexspecific characteristics of the sample were obtained by simple tabulations and descriptive statistics. Differences for continuous variables among the subgroups were assessed by one-way ANOVA. Multiple pairwise comparisons were made with a post hoc Scheffe test; the significance level of the p-value was reduced to 0.005 .

\section{Results}

After random selection of 100 subjects in accordance with the age-sex distribution of the initial sample, accelerometer measurements were performed. Because of too long non-wear period 7 subjects were excluded. The final sample included 39 males and 54 females. The percentage of physically active subjects were determined in accordance with the 3 criteria mentioned above, in males and females, in 4 age groups (25-34 y.o., 35-44 y.o., 45-54 y.o. and $\geq 55$ y.o.) and among obese (BMI $\left.\geq 30 \mathrm{~kg} / \mathrm{m}^{2}\right)$ and non-obese $\left(\mathrm{BMI}<30 \mathrm{~kg} / \mathrm{m}^{2}\right)$ subjects — results are presented in Table 1.

Mean number of steps/week and average time in 10-minute MVPA bouts/week were calculated among physically active and inactive subjects (according to steps/day criteria and time in 10-min MVPA bouts criteria) and in subjects of different occupational categories. Surprisingly, no significant differences in number of steps or MVPA time were found across the occupational categories - results are shown in Table 2.

The seven-day wear time protocol provided the possibility to discriminate workweek and weekend days. In Figure 1, it can clearly be seen that physically active subjects (according to 10 -min bouts criteria) are more active during the whole week (35-53 minutes of MVPA/day, including the weekend). The non-active group accumulated about 8-12 MVPA minutes/day, and they are even less active on weekends.

Comparing time in 10-min bouts of MVPA on workweek and weekend for every participant, physically active subjects (Fig. 2) have more balanced PA

Table 1. Proportions of physically active subjects according to different optimal physical activity (PA) criteria depending on age, gender and anthropometric features

\begin{tabular}{|c|c|c|c|c|}
\hline & $\mathrm{N}$ of subjects & $>10000$ steps/day & $\begin{array}{c}>300 \mathrm{~min} / \text { week } \\
\text { in MVPA }\end{array}$ & $\begin{array}{c}>150 \mathrm{~min} / \text { week in } 10-\mathrm{min} \\
\text { bouts of MVPA }\end{array}$ \\
\hline All & 93 & $57 \%$ & $70 \%$ & $37 \%$ \\
\hline \multicolumn{5}{|l|}{ Age } \\
\hline $25-34$ y.o. & 21 & $52 \%$ & $70 \%$ & $41 \%$ \\
\hline $35-44$ y.o. & 17 & $46 \%$ & $69 \%$ & $61 \%$ \\
\hline $45-54$ y.o. & 26 & $40 \%$ & $64 \%$ & $32 \%$ \\
\hline$\geq 55$ y.o. & 29 & $35 \%$ & $82 \%$ & $32 \%$ \\
\hline$p$ between age groups & & 0.53 & 0.51 & 0.61 \\
\hline \multicolumn{5}{|l|}{ Sex } \\
\hline Males & 39 & $48 \%$ & $63 \%$ & $39 \%$ \\
\hline Females & 54 & $38 \%$ & $78 \%$ & $38 \%$ \\
\hline $\mathrm{p}$ males vs. females & & 0.24 & 0.28 & 0.54 \\
\hline \multicolumn{5}{|l|}{ BMI } \\
\hline$<30 \mathrm{~kg} / \mathrm{m}^{2}$ & 57 & $44 \%$ & $69 \%$ & $38 \%$ \\
\hline$\geq 30 \mathrm{~kg} / \mathrm{m}^{2}$ & 36 & $36 \%$ & $76 \%$ & $36 \%$ \\
\hline p obese vs. non-obese & & 0.33 & 0.38 & 0.53 \\
\hline
\end{tabular}

MVPA - moderate- to vigorous-intensity physical activity; BMI — body mass index 
Table 2. Number of steps and time in 10-min bouts of moderate- to vigorous-intensity physical activity (MVPA) during 1 week in subjects with different physical activity (PA) status and occupation

\begin{tabular}{|c|c|c|c|c|}
\hline & Steps count & SE & Time 10 min bouts & SE \\
\hline$<10000$ steps/day $(\mathrm{n}=28)$ & 51027.46 & 2422.54 & 92.26 & 10.32 \\
\hline$\geq 10000$ steps/day $(n=65)$ & 95215.21 & 5012.62 & 230.14 & 22.87 \\
\hline p & $<0.001$ & & $<0.001$ & \\
\hline$<150$ min in 10 min bouts $(n=40)$ & 54321.43 & 2625.55 & 65.74 & 5.96 \\
\hline$\geq 150$ min in 10 min bouts $(n=53)$ & 94108.03 & 5850.78 & 285.19 & 14.44 \\
\hline $\mathbf{p}$ & $<0.001$ & & $<0.001$ & \\
\hline Intellectual group $(n=29$ ) & 78503.58 & 9829.99 & 176.09 & 31.07 \\
\hline Manual group $(n=33)$ & 78204.43 & 6034.73 & 152.71 & 36.24 \\
\hline Average group $(\mathrm{n}=31)$ & 64170.78 & 4789.04 & 140.78 & 19.60 \\
\hline p & 0.72 & & 0.43 & \\
\hline
\end{tabular}

SE - standard error

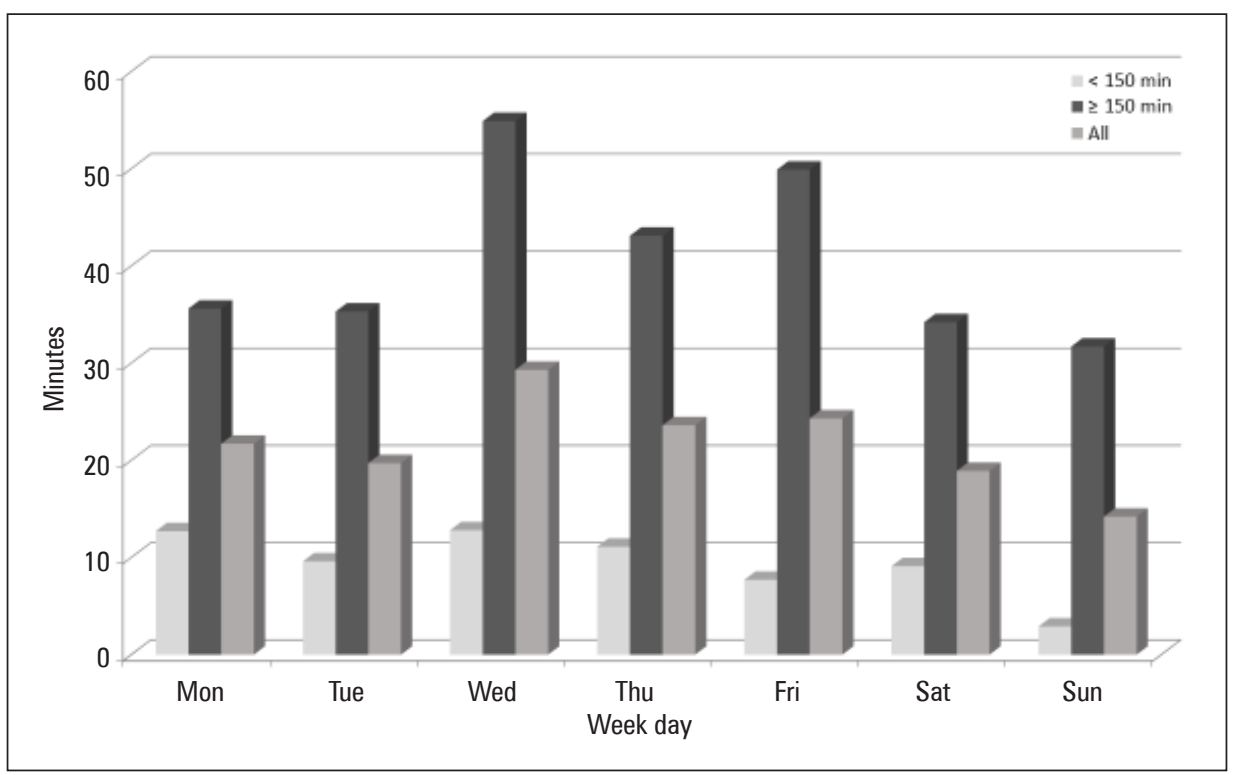

Figure 1. Seven-day pattern of time in 10-min bouts of moderate- to vigorous-intensity physical activity (MVPA) in subjects with different physical activity (PA) status

profile during whole week, i.e. $42 \%$ of them have same levels of physical activity on workweek and weekends, $42 \%$ are more active during workweek and $16 \%$ - on weekend.

Only 13\% of inactive participants have balanced MVPA bouts during the week, $70 \%$ of them are mainly active during workweek and $17 \%$ - on weekend (Fig 3).

Describing a typical day of physically active and physically inactive subjects (according to 10-min MVPA bouts criteria) in both groups we can see the same peaks of MVPA at 8.00-9.00 and 18.00-19.00 time, which are the periods of typical home-to-work and work-to-home transportation. Some optimal PA subjects have MVPA episodes during work time (at 12.00-13.00 and at 15.00) independently of type of occupation, but from Figure 4 it can clearly be seen, that subjects who are physically active are more active on their transportation time and during whole work time.

\section{Discussion}

This study provides first Russian results of 7-day objective measurement of physical activity duration and intensity using triaxial accelerometers. According to all three criteria of optimal PA level, no signifi- 


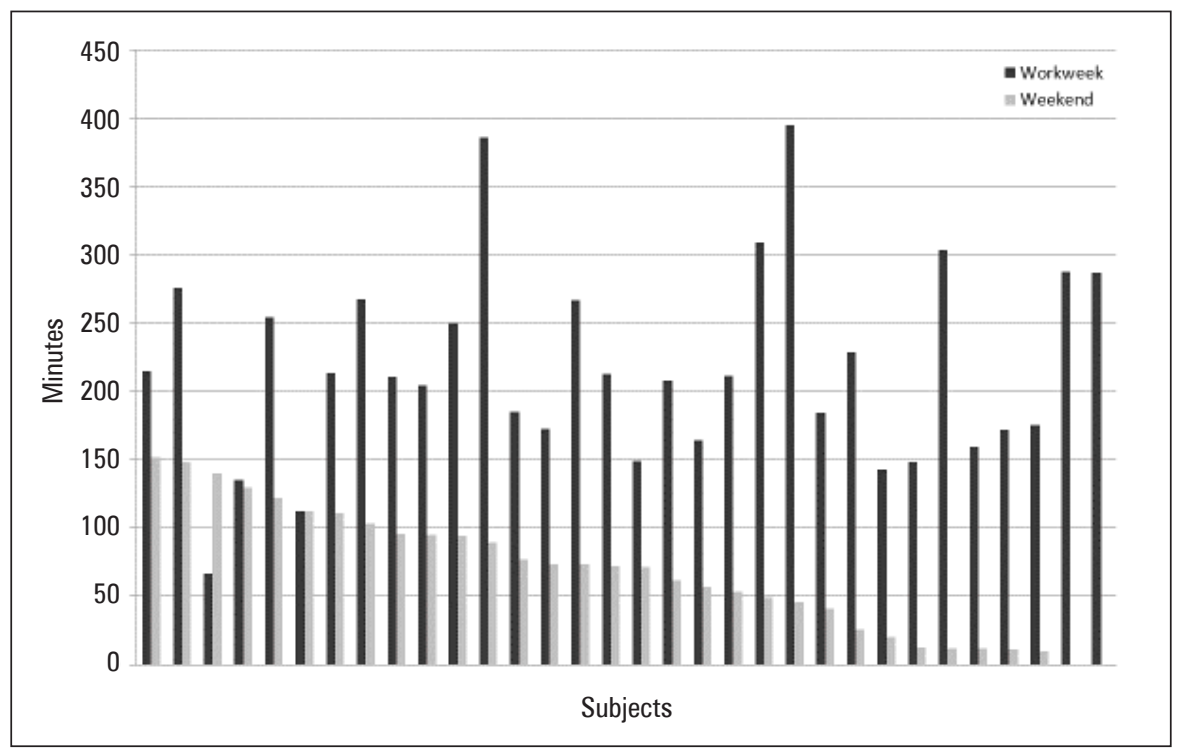

Figure 2. Comparison of time in 10-min moderate- to vigorous-intensity physical activity (MVPA) bouts on workweek and weekend in subjects with adequate level of physical activity (PA)

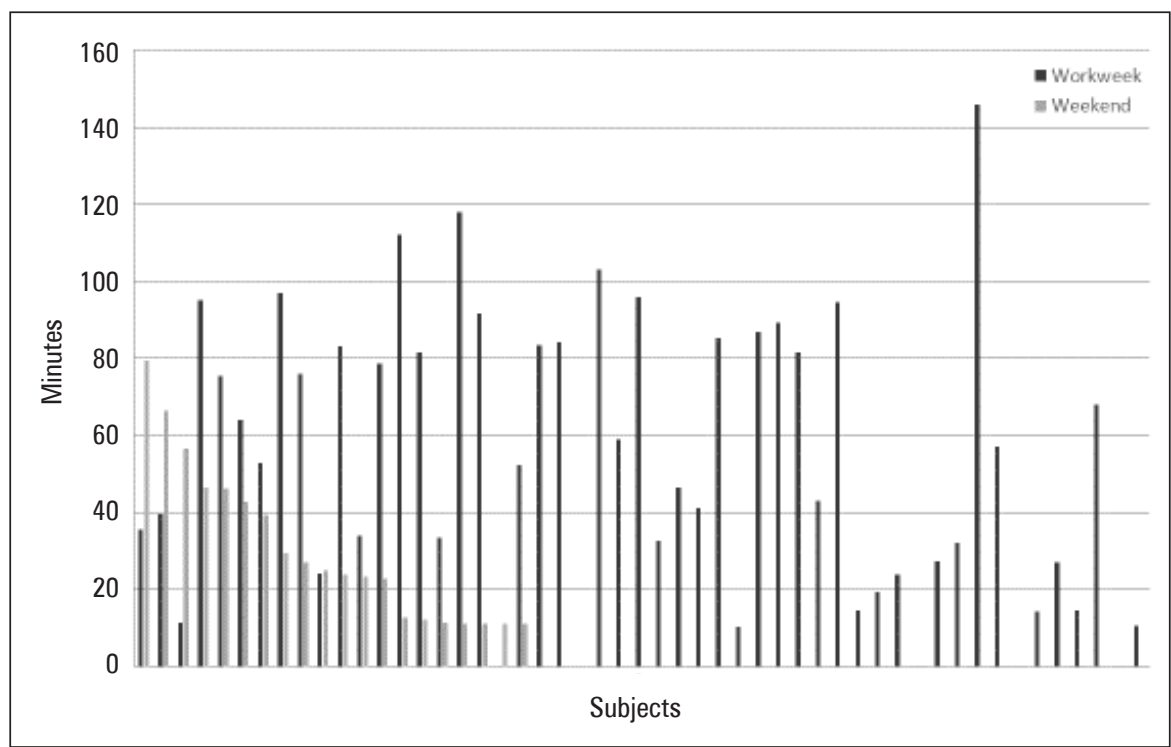

Figure 3. Comparison of time (minutes) in 10-min moderate- to vigorous-intensity physical activity (MVPA) bouts on workweek and weekend in subjects with non-optimal level of physical activity (PA)

cant differences were found between four age groups, males and females or obese and non-obese subjects. Occupation also showed no influence on the physical activity status. Main MVPA-providing period was morning and evening transportation time, and physical inactive behavior may be associated with an increased use of private or public cars on 15-min walking distances.

Since the mid-1980s, there has been a steady increase in data concerning association of physical activity levels with risk of non-communicable diseases, such as type 2 diabetes, obesity, and cardiovascular disease [8]. Previous publications have shown that self-reported physical activity time and intensity may be completely inaccurate [9]. Nowadays, it is clear that movement sensors, such as pedometers and accelerometers, may overcome most of the problems related to self-report [10]. While pedometers are specifically designed to measure features of walking behaviors such as total steps taken per day [11], accelerometer-based physical activity monitors allow researchers to track frequency, in- 


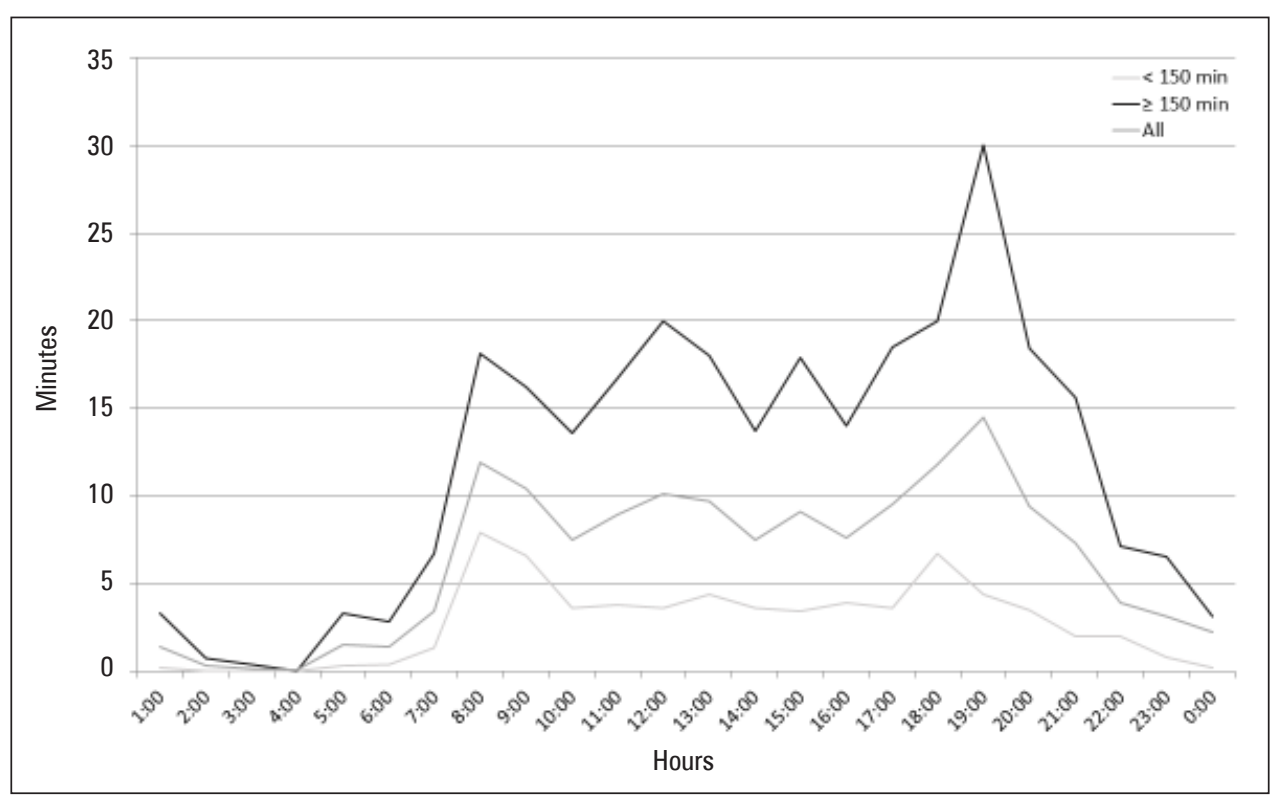

Figure 4. Physical activity (PA) (in minutes) of moderate- to vigorous-intensity physical activity (MVPA) during average 24-hour period in subjects with different PA levels

tensity, and duration of activity [12]. Prior to the development of triaxial accelerometers, uniaxial accelerometers were used to measure accelerations that occurred within the vertical plane [13]. Triaxial accelerometers capture movement in all 3 orthogonal planes. As a result, these devices provide the opportunity to capture many more activities than uniaxial accelerometers; thus, in comparison with uniaxial instruments, the output from triaxial devices tends to have higher correlations with energy expenditure [14]. Therefore, using of triaxial accelerometers may help to avoid bias of self-reported physical activity measurement, give new information on correlations between physical activity level and risk factors for non-communicable diseases and help to update modern PA recommendations.

According to $\mathrm{WHO}$ recommendations, NCD-risk reductions routinely occur at levels of at least 150 minutes of moderate-intensity activity per week, achieved through accumulation of bouts of 10 or more consecutive minutes [15]. But it is important to recognize that the current recommendations [8, 15] are based on epidemiological data concerning association between self-reported physical activity and health outcomes $[4,8,15]$. Because of low consistency of self-reported and objectively measured physical activity levels in big studies, like NHANES in USA [16], the need to change PA recommendations was discussed. According to PA diary and actigraphy with triaxial accelerometers, performed in our study, it can clearly be seen, that main physical activity is performed with walking, as in other studies
[16]. Analyzing 10-min MVPA bouts during whole week, it was shown, that the majority of subjects with optimal (> $150 \mathrm{~min}$ of MVPA/week) level of PA have a balanced workweek-weekend profile, so this participants are active every day independently of sex, BMI or occupation. On the other hand, inactive subjects have lower levels of MVPA during workweek and even less activity on weekend. According to PA diary, filled in during PA monitoring by every participant, it may be explained mainly with use of personal or public cars for transportation and lack of compensating self-trainings on weekends. Physical activity profile during an average day is similar in both active and non-active subjects - there are two main PA peaks in morning and evening time, describing episodes of home-to-work transportation. In the physically active group, average time in MVPA on these peaks is 15 minutes in the morning and 20-30 minutes in the evening - walking time from place of work to metro or other destination point, according to PA diaries. There are the same peaks in non-active group, but much lower - about 7 minutes, mainly caused by using cars on most days, according to PA diary.

The results of triaxial PA-monitoring in the random sample of Saint-Petersburg inhabitants demonstrate, that 150-min MVPA goal can easily be achieved by increasing walking time in two natural home-to-work and work-to-home transportation peaks on workweek and some additional exercises on weekend. According to PA-monitoring and diary information, main reason for lack of physical activ- 
ity cannot be found in the type of occupation, sex or age, but in the use of cars in morning and evening transportation time, instead of walking.

\section{Competing interests}

No potentially competing interests are declared.

\section{Financial disclosure}

These authors have no support or funding to report.

\section{Ethics statement}

The study has been approved by the Ethics Committee of Almazov National Medical Research Centre, Saint Petersburg, Russia. Written informed consent was provided by all patients. All medical data used in this study have been anonymized.

\section{References}

1. World Health Organization. Global health risks: mortality and burden of disease attributable to selected major risks. WHO, Geneva 2009.

2. Physical Activity Guidelines Advisory Committee (PAGAC). Physical Activity Guidelines Advisory Committee Report. Department of Health and Human Services, Washington 2008.

3. Nocon M, Hiemann T, Müller-Riemenschneider F, et al. Association of physical activity with all-cause and cardiovascular mortality: a systematic review and meta-analysis. Eur J Cardiovasc Prev Rehabil. 2008; 15(3): 239-246, doi: 10.1097/HJR.0b013e3282f55e09, indexed in Pubmed: 18525377.

4. Sofi F, Capalbo A, Cesari F, et al. Physical activity during leisure time and primary prevention of coronary heart disease: an updated meta-analysis of cohort studies. Eur J Cardiovasc Prev Rehabil. 2008; 15(3): 247-257, doi: 10.1097/HJR.0b013e3282f232ac, indexed in Pubmed: 18525378.

5. Chen KY, Bassett DR. The technology of accelerometry-based activity monitors: current and future. Med Sci Sports Exerc. 2005; 37(11
Suppl): S490-S500, doi: 10.1249/01.mss.0000185571.49104.82, indexed in Pubmed: 16294112.

6. Scientific Organizing Committee of the ESSE-RF. Epidemiology of cardiovascular diseases in different regions of russia (ESSE-RF). The rationale for and design of the study. Prev Med. 2013; 6: 25-34.

7. Pate RR, Pratt M, Blair SN, et al. Physical activity and public health. A recommendation from the Centers for Disease Control and Prevention and the American College of Sports Medicine. JAMA. 1995; 273(5): 402-407, doi: 10.1001/jama.273.5.402, indexed in Pubmed: 7823386.

8. Physical Activity Guidelines Advisory Commitee Report. United States Department of Health and Human Services, Washington 2008: A1-A10.

9. Duncan GE, Sydeman SJ, Perri MG, et al. Can sedentary adults accurately recall the intensity of their physical activity? Prev Med. 2001; 33(1): 18-26, doi: 10.1006/pmed.2001.0847, indexed in Pubmed: 11482992.

10. Lagerros YT, Lagiou P. Assessment of physical activity and energy expenditure in epidemiological research of chronic diseases. Eur J Epidemiol. 2007; 22(6): 353-362, doi: 10.1007/s10654-0079154-x, indexed in Pubmed: 17599242.

11. Mathie MJ, Coster ACF, Lovell NH, et al. Accelerometry: providing an integrated, practical method for long-term, ambulatory monitoring of human movement. Physiol Meas. 2004; 25(2): R1-20, doi: 10.1088/0967-3334/25/2/r01, indexed in Pubmed: 15132305.

12. Plasqui $G$, Joosen $A M$, Kester $A D$, et al. Measuring free-living energy expenditure and physical activity with triaxial accelerometry. Obes Res. 2005; 13(8): 1363-1369, doi: 10.1038/oby.2005.165, indexed in Pubmed: 16129718.

13. Welk GJ. Physical activity assessments for health-related research. Human Kinetics Publishers, Inc, Champaign 2002: 16.

14. Hendelman D, Miller K, Baggett C, et al. Validity of accelerometry for the assessment of moderate intensity physical activity in the field. Med Sci Sports Exerc. 2000; 32(9 Suppl): S442-S449, doi: 10.1097/00005768-200009001-00002, indexed in Pubmed: 10993413.

15. World Health Organization. Global recommendations on physical activity for health. WHO, Geneva 2008.

16. Troiano RP, Berrigan D, Dodd KW, et al. Physical activity in the United States measured by accelerometer. Med Sci Sports Exerc. 2008; 40(1): 181-188, doi: 10.1249/mss.0b013e31815a51b3, indexed in Pubmed: 18091006. 